\title{
LSGO: Link State aware Geographic Opportunistic routing protocol for VANETs
}

\author{
Xuelian Cai, Ying He, Chunchun Zhao, Lina Zhu and Changle Li*
}

\begin{abstract}
Robust and efficient data delivery in vehicular ad hoc networks (VANETs) with high mobility is a challenging issue due to dynamic topology changes and unstable wireless links. The opportunistic routing protocols can improve the reliability of routing by making full use of the broadcast characteristics and assist in data transmission through additional backup links. In this paper, we propose a Link State aware Geographic Opportunistic routing protocol (LSGO) which exploits a combination of geographic location and the link state information as the routing metric. The LSGO aims to improve the reliability of data transmission in a highly dynamic environment, which selects the forwarders and prioritizes them based on the vehicle's geographic location and the link's quality. We compare the performance of LSGO with GpsrJ + which removes the unnecessary stop at a junction and greedy traffic aware routing protocol (GyTAR) using network simulator ns-2. The simulation results show that it opens more nodes to participate in the opportunistic data forwarding and increases a connection's throughput while using no more network capacity than traditional routing. In the simulation, compared with other two protocols, when the number of vehicles and the average vehicle velocity increase, LSGO's packet dropping rate is reduced and the network throughput is improved.
\end{abstract}

Keywords: VANETs; Geographic opportunistic routing; Link state; Priority schedule

\section{Introduction}

Vehicular ad hoc networks (VANETs) [1] are first designed for safety applications; afterwards, a series of applications for increasing traffic efficiency and providing comfort to the vehicle's passengers are proposed. The network layer has received the most attention when working on VANETs. As a result, abundant routing protocols in such a network with differing objectives and for various specific needs have been proposed [2].

Existing routing protocols of VANETs fall into two major categories: topology-based and geographic routing. Topology-based routing [3-5] uses the information about links that exist in the network to perform packet forwarding. Since link information changes in a regular basis, topology-based routing suffers from routing breaks, so this kind of routing protocols is not suitable for VANETs. Geographic routing [6-15] uses neighboring location information to perform packet forwarding. In this kind of routing protocols, nodes are unnecessary to

\footnotetext{
* Correspondence: clli@mail.xidian.edu.cn

State Key Laboratory of Integrated Services Networks, Xidian University, Xi'an, Shaanxi 710071, China
}

maintain a topology map or exchange link state information or maintain established routes as they do in a conventional mobile ad hoc routing protocol. Therefore, geographic routing can better adapt to network size and topology changes.

Greedy forwarding is the most widely used strategy in geographic routing. The fundamental principle is that a node forwards its packet to its neighbor that is closest to the destination. But the forwarding strategy can fail if no neighbor is closer to the destination than the node itself, and through this way, we can get the next hop which is nearly located beyond the transmission range of the forwarder. In this case, the established link is unstable and the signal strength may be reduced, which may cause an increase of the packet dropping rate. As the packet is forwarded using this kind of links, the probability of packet transmission failure is great. So, it will spend more resources on retransmissions. As a result, the network throughput is declined and the end-to-end delay is prolonged.

To solve this problem, De Couto et al. proposed a new measure called the expected transmission count (ETX) 
[16], which is the predicted number of data transmissions required to send a packet over the source to the destination link, including retransmissions. The ETX is widely used in routing protocols for wireless multihop networks [17-20] since its goal is to find the paths with the higher throughput and the less expected total number of transmission [21]. The difficulty in using ETX in VANETs is that ETX does not consider the highly dynamic network environment, so we modified the ETX in this paper.

Although greedy forwarding strategy makes the hop transmission to the greatest extent close to the destination, the link is very unstable, which is because the two nodes at both ends of the link are located at the boundary of each other's communication range. So, another forwarding strategy opportunistic routing is proposed, which could improve the reliability of data transmission by making full use of the broadcast characteristics and assist in data transmission through additional backup links. It makes the packets have more opportunities to be received. In the existing opportunistic routing protocols, some take hop count as the routing metric, some pay more attention to the cost, some consider the distance to the destination to be the forwarding mechanism, and some care more about the energy. However, few of them take a combination of geographic location and the link state information into account. So, we proposed a Link State aware Geographic Opportunistic routing protocol (LSGO) which takes a combination of geographic location and the link state information as the forwarder selection mechanism. Firstly, we propose a candidate node set selection mechanism, which selects the forwarders based on the vehicle's geographic location and the link's quality. In our approach, the link's quality is measured using the enhanced ETX metric. Secondly, we put forward a priority scheduling algorithm which prioritizes the forwarders by timer-based scheduling method. This routing protocol can greatly improve the packet delivery ratio, ensuring data transmission reliability under a highly dynamic environment.

The rest of the paper is organized as follows: In Section 2, we will review the related work and introduce our motivation. Section 3 will present the details in the proposed LSGO scheme. The performance evaluations of the proposed scheme are presented in Section 4. Finally, Section 5 concludes the article.

\section{Related work}

To design a routing protocol is to propose a method, by which the current node could select the appropriate intermediate node as its next hop. So far, geographic routing protocols are widely used in VANETs. However, there are some problems in conventional geographic routing protocols. For example, greedy perimeter stateless routing's (GPSR's) [6] recovery mode has a problem called Baby Step Problem [22]. To solve this problem, greedy perimeter coordinator routing (GPCR) [7] is proposed. Packets are always greedily forwarded along the road from one junction to the other, which solves the Baby Step Problem in GPSR. However, even if a packet is forwarded along the street, it needs to stop at each junction node. GpsrJ + [8] removes the unnecessary stop at a junction while keeping the efficient planarity of topological maps. Recently, many researchers are concerned about opportunistic routing protocols since the initial work extremely opportunistic routing (ExOR) [18] aroused great repercussion. Opportunistic routing is a new routing strategy, from which it was proposed to be widespread concerned, only experienced a few years time. The biggest difference between opportunistic routing and traditional routing is that opportunistic routing does not use a fixed route, but the relay nodes self-select the next hop to send or not to be forwarded according to the routing protocol. This process continues until the destination node receives the packets. During each packet transmission, whether it is the source nodes that send or the relay nodes that forward, opportunistic routing makes the packet have more opportunities to be received than traditional routing. So, this type of routing protocol is called opportunistic routing, and its basic model is shown in Figure 1. Assume that the node $N_{i}$ wants to send a data packet to the destination node $N_{d}$, and the $N_{d}$ is outside of the effective transmission range of $N_{i}$. We define $C_{i}=\left\{N_{1}, N_{2}, \cdots, N_{n}\right\}$ as a candidate node set of node $N_{i}$, which is a subset of neighbor nodes and contains all the forwarders selected based on a candidate node selection strategy. $C_{i}$ is an ordered collection, and the order of the elements in the set is the same as the priority they forward the received data packets. Extending the concept of geographical routing, some opportunistic routing protocols are proposed in recent years. Biswas and Morris introduced the novel ExOR [18] protocol and showed that network nodes can

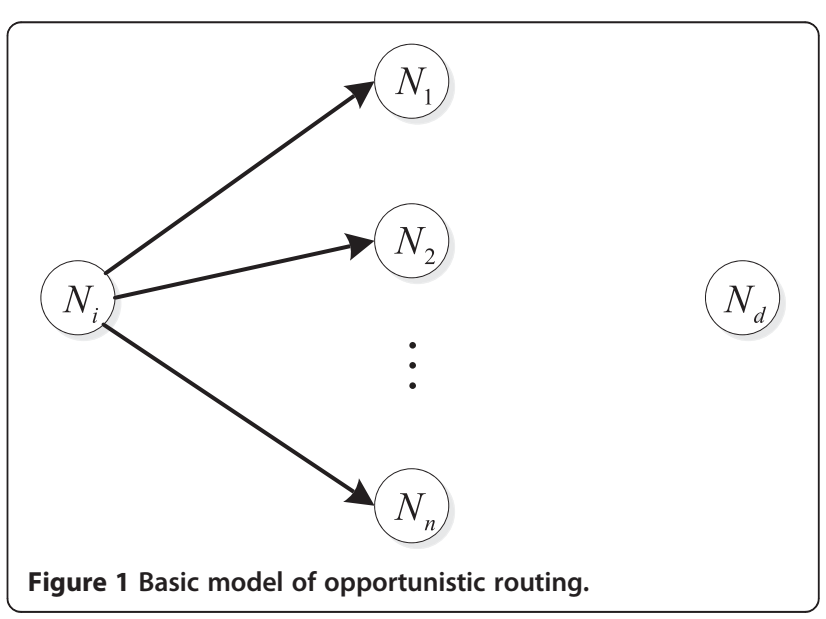


achieve better performance than the traditional routing protocols. ExOR does utilize the overhearing in wireless networks by including all nodes on the route to be intended next forwarder, but it still has some limitations. That is because only the nodes included in the forwarder list can participate in the opportunistic data forwarding and benefit from the broadcast nature to enhance the network performance. Chachulski et al. proposed the MORE [23] to address issues in ExOR and achieved high throughput in wireless networks. It enhances ExOR to further increase the spatial reuse in a single flow from source to destination via intra-flow network coding. However, when nodes have a malicious behavior, the adoption of such opportunistic routing protocols might reduce network throughput. Zhao and Cao [24] put forward a vehicle-assisted data delivery (VADD) routing protocol which is aimed at improving routing in disconnected vehicular networks by the idea of carry-andforward based on the use of predictable vehicle mobility. A vehicle makes a decision at a junction and selects the next forwarding path with the smallest packet delivery delay. A path is simply a branched road from an intersection. In the routing process, every node needed to know its geographical location and the static electronic map and depended on the weights of every road to make choices. Simulation indicated that VADD had lower transmission delay compared with other routing protocols. Leontiadis and Mascolo [25] proposed a geographical opportunistic routing (GeOpps) algorithm. It takes advantage of the suggested routes of the vehicles' navigation system to select vehicles that are likely to move closer to the final destination of a packet. It calculates the shortest distance from the packet's destination to the nearest point of the vehicles' path and estimates the arrival time of a packet to the destination. During the travel of vehicles, if there is another vehicle that has a shorter estimated arrival time, the packet will be forwarded to that vehicle. The process repeats until the packet reaches the destination. GeOpps requires navigation information to be exposed to the network; thus, privacy such as the vehicle's whereabouts might be an issue. A global approach is proposed in the routing protocol energy-efficient opportunistic routing (EEOR) of Mao et al. [26], which selects a route that is expected to use the lowest energy among all the routes between source and destination to deliver packets. EEOR does not consider the link quality between adjacent vehicles which may lead to these vehicles appearing on disjoint paths to the destination. Dubois-Ferriere et al. proposed a protocol called the least-cost anypath routing (LCAR) [27], which selects anypath but not the shortest path, in order to reduce retransmissions. Every node selects its forwarder list by taking all possible subsets of its neighbor set into account first and then calculating the relay cost from each of the subsets to the destination. The sum of the relay set cost is the total cost from the selector. The subset that has the minimum total cost of the selector is chosen as the relay set. The problem of LCAR is that it may select unnecessary large sets. Wang et al. [28] put forward a local cooperative relay strategy for opportunistic data forwarding, which proposes the step to choose the best local relay node from many candidate nodes but require no inner communication between them. The best local relay node is called helper-node, and it is selected just when it is needed. However, the shortcoming of the method is that the forwarder list is no longer sufficient to regulate the sequence of data transmissions, so further research is needed on how to reduce the coordination overhead and depress the collisions. Mazumdar and Sairam [29] first analyze the cause of duplicate transmissions and then put forward a forwarder selection method called transmission-aware opportunistic ad hoc routing (TOAR) that addresses to minimize retransmissions. TOAR selects a few candidate nodes and prioritizes them by using a tree structure. The method helps in recognizing the primary candidate node which is the one that, during a transmission round, can carry data farthest to the destination node. In addition, TOAR can help in selecting another kind of candidate nodes which transmit packets missed out by the candidate. The strategy leads to a smaller candidate node list set and ends up in reducing retransmissions. A localized opportunistic routing (LOR) protocol is proposed in [30], which utilizes the distributed minimum transmission selection algorithm to partition the topology into several nested close-node-sets using local information. For a large-scale wireless network, LOR can locally select the optimal opportunistic routing with low overhead cost. The highlight of this method is that it makes a trade-off between the scalability caused overhead and the optimality of the candidate node lists. It reduces the control overheads but does not take the asymmetric wireless channels into account. A few of these protocols have considered the link state when they select the candidate relay nodes and prioritize their transmission. In this paper, we propose the LSGO routing protocol, which takes a combination of opportunistic routing and link state information into account when we design the candidate node set selection mechanism and the priority scheduling algorithm. The proposed approach could be applied to large-scale wireless networks [31,32].

\section{Link State aware Geographic Opportunistic routing protocol}

We propose an opportunistic routing called Link State aware Geographic Opportunistic routing protocol (LSGO) which takes a combination of geographic location and the link state information as the forwarder selection 
mechanism. The protocol aims to ensure a highly dynamic network packet delivery rate and improve the reliability of data transmission. Besides, it also aims to reduce the number of transmissions (including retransmissions) and the transmission delay. The protocol mainly includes three parts, namely, the estimation of link quality, candidate node set selection mechanism, and priority scheduling algorithm.

\subsection{The estimation of link quality}

ETX [16] is based on the expected number of transmissions (including retransmissions) to select the next hop, and the aim is to minimize the end-to-end number of transmissions, thus saving bandwidth. The ETX of a path is the sum of the ETX value of each link on this path. Each node broadcasts probe packets periodically. After a certain time interval, two adjacent nodes calculate the probe packet delivery rate $d_{f}$ and $d_{r}$ in two directions (one for probe packet transmission and the other for ACK acknowledgment packet transmission). So, the expected probability of a successful transmission is $d_{f} \times d_{r}$. Since every time we send a data packet can be considered as a Bernoulli trial, ETX is calculated as

$$
\mathrm{ETX}=\frac{1}{d_{f} \times d_{r}}
$$

However, the ETX metric does not specifically consider the mobility in VANETs. In LSGO, we improve the ETX to adapt to the network that is highly dynamic. There are two major improvements: the measurement of the link transmission rate and the calculation of ETX.

In LSGO, each node broadcasts a Hello packet periodically, and we use the Hello packets to measure the link transmission rate. To calculate the ETX of a link, each node should record $t_{0}$ which means the time when the first Hello packet is received and the number of packets it has received from the neighbor during the last $w$ seconds. Then, according to the interval between $t_{0}$ and the current time $t$ and the window $w$, the link transmission rate $r(t)$ is

$$
r(t)=\left\{\begin{array}{l}
\operatorname{count}\left(t_{0}, t\right), \quad 0<t-t_{0}<1 \\
\frac{\operatorname{count}\left(t_{0}, t\right)}{\left(t-t_{0}\right) / \tau}, \quad 1 \leq t-t_{0}<w \\
\frac{\operatorname{count}(t-w, t)}{w / \tau}, t-t_{0} \geq w
\end{array}\right.
$$

The denominator is the number of Hello packets that should have been received during the window, and $\tau$ represents the broadcast interval of the Hello packet. Count $\left(t_{0}, t\right)$ is the number of Hello packets received during $t-t_{0}$. As can be seen from the formula, there are three situations in terms of the difference between $t-t_{0}$ and window $w$. (1) $0<t-t_{0}<1$, in this case, the packet delivery rate is the number of Hello packets received from $t_{0}$ to $t$. (2) $1 \leq t-t_{0}<w$, the packet delivery probability in this condition is the number of Hello packets received from $t_{0}$ to $t$ divided by the length of this period. (3) $t-t_{0} \geq w$, in this situation, the calculation is the same as the calculation in the ETX metric.

In LSGO, we do not consider the asymmetry of the link and only use the one-way transmission rate to calculate the link ETX. Assuming that the one-way transmission rate is $r(t)$, then the link ETX is

$$
\mathrm{ETX}=\frac{1}{r^{2}(t)}
$$

\subsection{Candidate node set selection mechanism}

LSGO's main objective is to use opportunistic routing to ensure VANET transmission reliability, while reducing the number of transmissions, and therefore, the selection of the candidate node set needs to ensure that the number of backup links can provide the required delivery rate. Seen from the estimation of link quality, each node can calculate the link transmission rate $r(t)$ of all links between itself and all its neighbors. The candidate nodes can be selected by the link transmission rates of the links that are formed by the sending node to its neighbors. As shown in Figure 2, $r_{1}(t)$ and $r_{2}(t)$ are the transmission rates of the source node $\mathrm{S}$ to its two candidate relay nodes $\mathrm{X}$ and $\mathrm{Y}$. Then, the probability that $\mathrm{S}$ sends data to the next hop successfully is $1-\left(1-r_{1}(t)\right)\left(1-r_{2}(t)\right)$.

Here is how the candidate node set selection mechanism works. For node $\mathrm{S}$, the current time $t$, the number of neighbor nodes is $N . r_{i}(t)(1 \leq i \leq N)$ is the transmission rate of the link that is formed by $S$ to its neighbor node $i$, and $d_{i}(t)(1 \leq i \leq N)$ represents the distance from the destination to node $i . S(t)$ is the distance from the

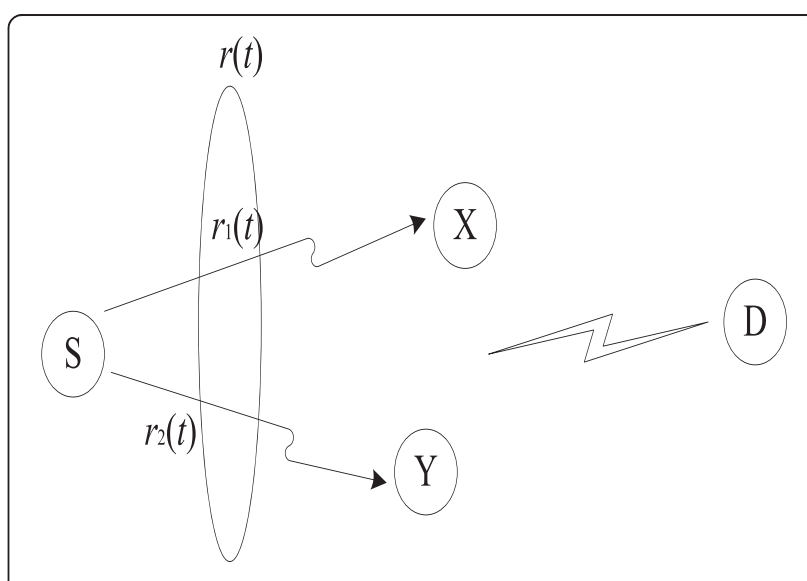

Figure $2 \mathrm{~S}$ sends packets to $\mathrm{D}$ via the relay node $\mathrm{X}$ or $\mathrm{Y}$. 
current node to the destination, and $r$ is the required data delivery rate of a single link. If the number of candidate nodes is $n$, then $n$ should satisfy the following conditions:

$$
\begin{aligned}
& 1-\prod_{i=1}^{n}\left(1-r_{i}(t)\right) \geq r \\
& d_{1}(t)<d_{2}(t)<\ldots<d_{n}(t)<d_{n+1}(t)<\ldots d_{N}(t) \\
& d_{n}(t)<S(t)
\end{aligned}
$$

That is, for the current node, the nodes in the candidate node set are the first $n$ neighbors nearest to the destination. In addition, the distances from these $n$ nodes to the destination are less than $S(t)$. Note that if the network is sparse, it may result in a situation in which those $n$ nodes cannot satisfy the condition $1-\prod_{i=1}^{n}\left(1-r_{i}(t)\right) \geq r$. At this time, only if the distance from the neighbor node to the destination is less than $S(t)$, the neighbor node is the candidate node.

The sending node would record the candidate nodes' IDs and their priority numbers in the packet header after it selected the candidate node set. Since the number of candidate nodes $n$ is dynamic, the size of the packet header is changing with it. If the network environment is good, the link between any two nodes is relatively stable, so the value of $n$ and the packet header is small, which means that the overhead is small. On the contrary, if $n$ and the packet header are large, then the overhead is large, too. The priority scheduling algorithm will be introduced in the next section.

\subsection{Priority scheduling algorithm}

LSGO uses timer-based priority scheduling algorithm, in which the highest priority node sends the packet firstly. For other candidate nodes, if they hear a higher-priority node send a packet, they would not process the packet; if the timer expires and a higher-priority node is not transmitting, they would begin to send the packet. The timer-based scheduling algorithm is simple and easy to implement and has no additional control overhead. However, the disadvantage is that it would introduce waiting time, thereby increasing the end-to-end transmission delay. Another shortcoming is that it may cause duplicate packet transmission, because the nodes in the candidate node set may not hear each other. But in VANETs, the packet passes along roads, and the road width is far less than the transmission range; in addition, the nodes that are selected by the candidate node set selection mechanism are located on one side of the current node, so all candidate nodes could hear each other from the distance perspective and duplicate transmission exists rarely in VANETs. An efficient scheduling algorithm should minimize the waiting time, which can be achieved in two ways: firstly, by assigning node priorities correctly, so that the optimal forwarding node has the highest priority and the higher-priority node has a better forwarding advantage, thus increasing the probability of selecting a higher-priority node that forwards packets and reducing the number of failed transmission, and secondly, by setting a reasonable waiting time for each node, which makes the low-priority node forward packets immediately after the high-priority node failed, thereby reducing the waiting time between the candidate nodes.

In LSGO, when the current node assigns the priority for a candidate node, it considers the distance from the candidate node to the destination, and the ETX of the link formed by the current node and the candidate node. There are two reasons for doing like this: on the one hand, selecting the candidate node that makes the greatest extent close to the destination as the forwarding node can reduce the transmission hops. On the other hand, the candidate node with a small ETX (minimum is 1) can increase the probability of successful reception. For candidate node $i$, its priority is obtained by

$$
\frac{D_{s d}-D_{i d}}{E T X_{i}^{2}}
$$

$D_{s d}$ is the distance between the current node and the destination. $D_{i d}$ is the distance from candidate node $i$

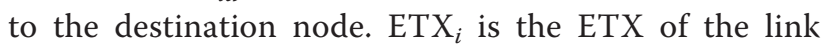
that is formed by the current node and candidate node $i$. $D_{s d}-D_{i d}$ indicates the geographic distance a packet can advance towards the destination. However, due to link loss, to be successfully forwarded to node $i$, a packet needs to be transmitted ETX $\mathrm{E}_{i}$ times on average. Therefore, $\left(D_{s d}-D_{i d}\right) / \operatorname{ETX}_{i}$ is the expected advance that a packet can make towards the destination through one transmission if it chooses node $i$ as the next hop.

Passing by a link of low transmission rate will increase the probability of data transmission failure, so we divide the square of ETX in Equation 7. If candidate node $i$ does not receive data correctly, another candidate node whose priority is lower than $i$ will transmit the data, thus introducing additional waiting time. If two nodes have the same expected advance that a packet can make towards the destination through one transmission, the node whose ETX is smaller should be set a high priority.

The sending node will calculate each candidate node's value according to Equation 7 as soon as it finishes selecting all the candidate nodes and assign priorities for candidate nodes in accordance with the calculation results. The node which has the maximum calculation result is assigned the highest priority; on the contrary, the node 
which has the minimum calculation result is assigned the lowest priority. The highest priority node sends a packet directly when it receives the packet, while the lower priority nodes need to set a timer. If the timer expires and a higher-priority node is not transmitting, they would begin to send the packet. Only by setting a reasonable overdue time for the timer can both reduce delay time and avoid duplication of transmission.

The network delay is defined as the time from a node receiving a packet to send it completely, and it consists of four parts: the processing delay, queuing delay, transmission delay, and propagation delay. Since we do not consider the network load, which means not considering the queuing delay, the network delay consists of three parts. Assuming that the total time of these three parts is $T$, if the node priority is $i$, the timer should be set to $(i-1) T$. In our simulation, the packet size is set to 512 bytes. The protocol in MAC layer is 802.11, in which the channel rate is $2 \mathrm{Mbps}$. So, the transmission delay is equal to $512 \times 8$ bits / $2 \mathrm{Mbps}=0.002048 \mathrm{~s}$. The radio wave propagation velocity in air is equal to the speed of light, namely, $3 \times 10^{8} \mathrm{~m} / \mathrm{s}$. However, the distance between two vehicles who can communicate with each other directly is less than $250 \mathrm{~m}$. So, the propagation delay is equal to $250 \mathrm{~m} / 3 \times 10^{8} \mathrm{~m} / \mathrm{s}=0.83 \times 10^{-6} \mathrm{~s}$, and it can be ignored. Through doing multiple times of simulation and analyzing the trace files, we can get the processing delay which is approximately $0.001 \sim 0.002 \mathrm{~s}$. Therefore, based on the above analysis, we can conclude that $T$ is about $0.004 \mathrm{~s}$.

\section{Simulation results and evaluation}

In this section, we study the performance of LSGO by running a computer simulation with network simulator ns-2 (version 2.34) [33]. GPSR is the most fundamental and classic geographic routing protocol, and it first proposes the greedy forwarding strategy, which is the most widely used strategy in VANETs. In addition, GPSR is the basis for most of the geographic routing protocols and often used as the comparison protocol. But its recovery mode has a problem called Baby Step Problem. To solve this problem, GPCR is proposed. Packets are always greedily forwarded along the road from one junction to the other, which solves the Baby Step Problem in GPSR. However, even if a packet is forwarded along the street, it needs to stop at each junction node. GpsrJ + [8] removes the unnecessary stop at a junction while keeping the efficient planarity of topological maps, improving GPCR to better adapt to the VANETs in a city scenario. It manages to increase the packet delivery ratio of GPCR and reduces the number of hops in the recovery mode compared to GPSR. So, we choose GpsrJ + as one of the contrast protocols. The greedy traffic aware routing protocol (GyTAR) [11] improves the greedy strategy that tries to mimic the shortest path routing by taking into account the road connectivity. A score is given to each neighboring junction considering the traffic density and their distance to the destination. It is good at finding robust routing in a city environment. Since the scenario in our simulation is also an urban scenario, we take GyTAR as another comparison protocol.

\subsection{Simulation settings}

The simulation scenario parameters are shown in Table 1. We set the road topology as shown in Figure 3 to build the scenario: size 2,500 $\mathrm{m} \times 1,500 \mathrm{~m}$, urban environment, roads are bidirectional. The transmission range of each vehicle is set to $250 \mathrm{~m}$. When the number of vehicles is the independent variable, there are 100 to 200 vehicles randomly distributed on the roads at the beginning of the simulation. Each vehicle's velocity ranges from 10 to $20 \mathrm{~m} / \mathrm{s}$. When the vehicle's velocity is the independent variable, there are 100 vehicles randomly distributed on the roads, and the vehicle's average velocity ranges from 9 to $24 \mathrm{~m} / \mathrm{s}$. The map is generated by the vehicular mobility model generator VanetMobiSim [34]. The propagation model used in the simulation is the two-ray ground model. The simulation time is $150 \mathrm{~s}$, and each simulation running contains ten random source-destination pairs. Each source node sends packets at the rate of $2 \mathrm{Mbps}$ with a packet size of 512 bytes. The Hello interval and window size $w$ are set to 1 and $10 \mathrm{~s}$, respectively. We evaluate the performance of the protocols by four metrics: (1) network throughput, (2) packet dropping rate, (3) end-to-end delay, (4) overhead.

\subsection{Results and analysis}

As shown in Figure 4, we compare the performance of the three protocols in terms of network throughput. The

\section{Table 1 Simulation parameters}

\begin{tabular}{lc}
\hline Parameters & Default value \\
\hline Simulation area & $2,500 \mathrm{~m} \times 1,500 \mathrm{~m}$ \\
Mobility model & VanetMobiSim \\
Transmission range & $250 \mathrm{~m}$ \\
Number of vehicles/vehicle velocity & $100 \sim 200 / 10 \sim 20 \mathrm{~m} / \mathrm{s}$ \\
Number of vehicles/vehicle velocity & $100 / 9 \sim 24 \mathrm{~m} / \mathrm{s}$ \\
MAC protocol & IEEE $802.11 \mathrm{DCF}$ \\
Channel rate & $2 \mathrm{Mbps}$ \\
Packet size & $512 \mathrm{bytes}$ \\
Number of CBR connections & 10 \\
CBR interval & $0.5 \mathrm{~s}$ \\
Hello interval & $1 \mathrm{~s}$ \\
Window size $W$ & $10 \mathrm{~s}$ \\
Simulation time & $150 \mathrm{~s}$ \\
\hline
\end{tabular}




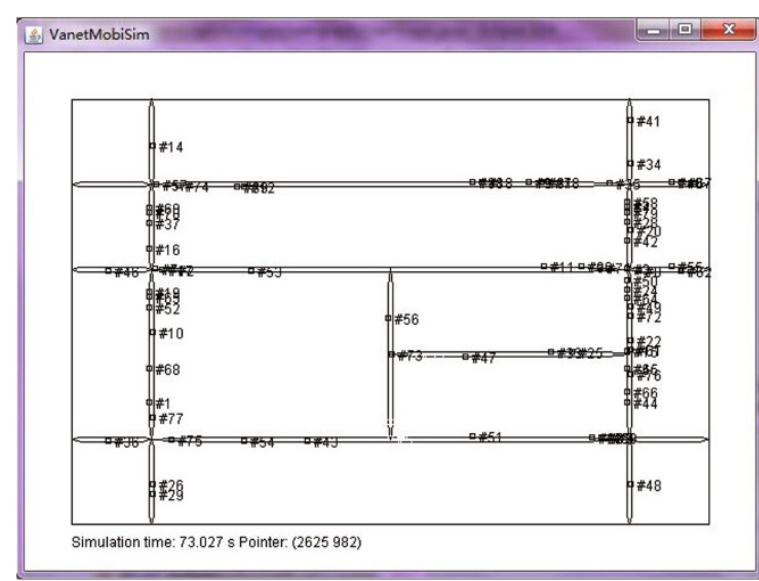

Figure 3 Simulation scenario.

network throughput is defined as the number of bits successfully transmitted per second in the network. In detail, the network throughput of GpsrJ + ranges from 595 to $1,356 \mathrm{kbps}$, while GyTAR increases from 624 to $1,602 \mathrm{kbps}$. However, we find that the network throughput of GpsrJ + and GyTAR is always lower than that of LSGO. LSGO's throughput ranges from 949 to $1,727 \mathrm{kbps}$. LSGO can achieve about 239 and 400 kbps average gain compared with GyTAR and GpsrJ+. LSGO selects a set of candidate nodes to forward the packet, so that it has a high probability of successful transmission of the packet, and consequently, the network throughput is improved.

Figure 5 illustrates the variation of packet dropping rate with the number of nodes. We define the packet dropping rate as the ratio of the number of packets that failed to be delivered to the destination to the number of the total packets generated in the simulation. As we can see, the average dropping rate of LSGO is about $37.3 \%$, while that of GyTAR and GpsrJ + is about $44.6 \%$

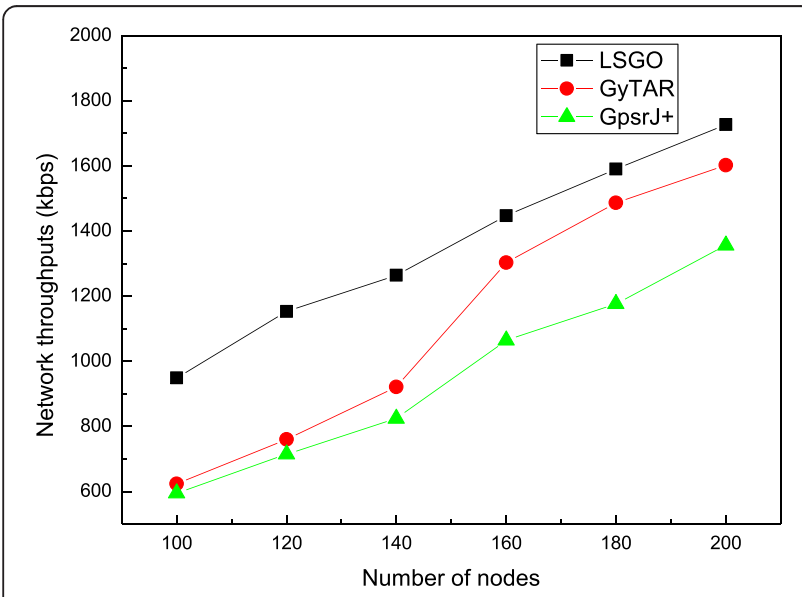

Figure 4 Network throughputs vs. number of nodes.

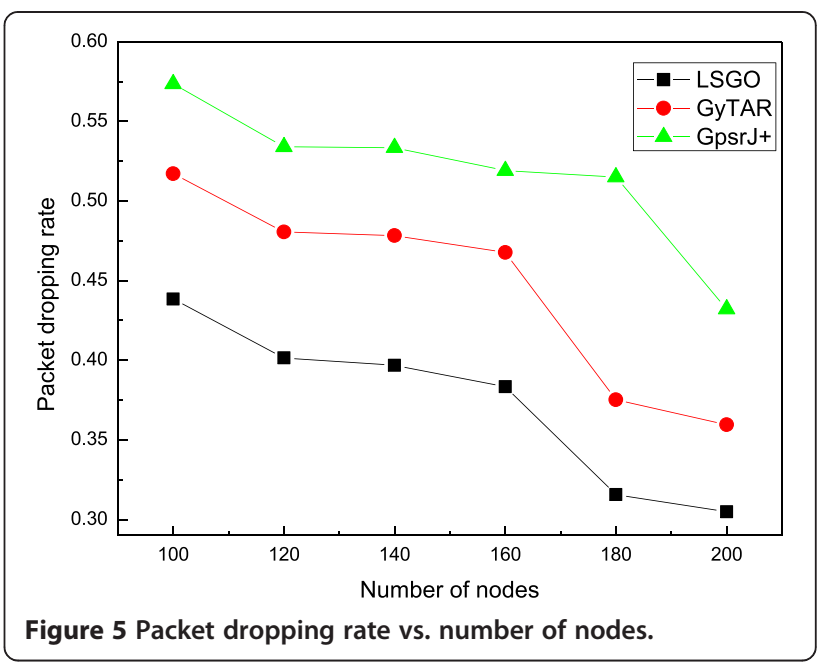

and $51.8 \%$, respectively. The set of candidate nodes and the relative high-quality links used in LSGO contribute to the improvement. In LSGO, we improve the greedy forwarding strategy by taking the link state into account, so the nodes that have a bad-quality link have low priorities to forward the packet. GyTAR and GpsrJ + choose the neighbor that is closest to the destination as the next hop, so the next hop is very likely located in the boundary of the forwarder's transmission range, and the links suffer a high packet dropping rate due to the channel fading. Therefore, the packet dropping rate achieved by GyTAR and GpsrJ + is higher than that by LSGO.

Figure 6 shows the performance of the end-to-end delay. The end-to-end delay is defined as the average amount of time spent by the transmission of a packet that is successfully delivered from the source to the destination. We can learn that the average delay of GpsrJ + is nearly 0.02 s. For GyTAR, the delay reaches to

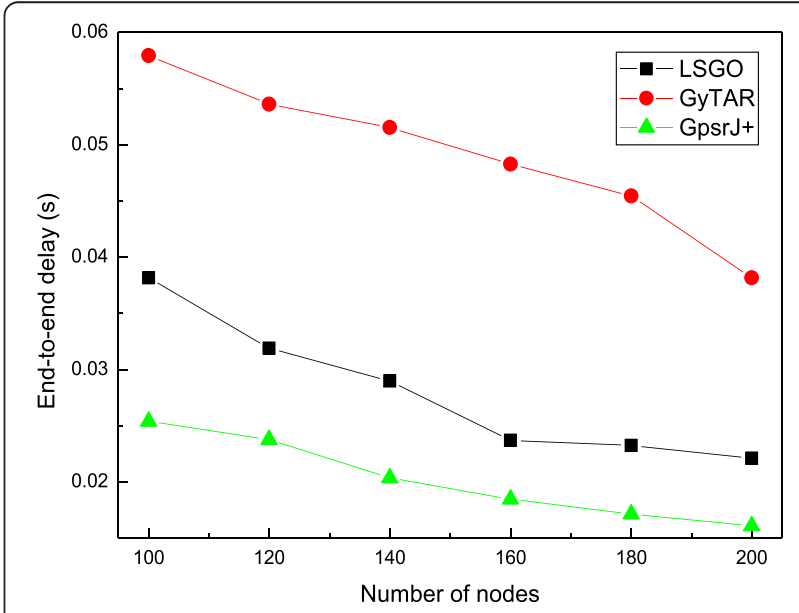

Figure 6 End-to-end delays vs. number of nodes. 
$0.058 \mathrm{~s}$ when there are 100 nodes in the network, and the minimum delay is $0.038 \mathrm{~s}$ when there are 200 nodes in the network. The delay of LSGO achieves $0.021 \mathrm{~s}$ average gain compared with GyTAR. This superiority is due to the reason that LSGO uses opportunistic routing to ensure VANET transmission reliability, while reducing the number of transmissions. LSGO takes the link quality into account when it chooses the next hop and selects a set of candidate nodes to forward the packet. The time needed to retransmission is saved, and thus, the end-toend delay is shortened, while in GyTAR, a considerable time would be wasted for retransmission.

Figure 7 reflects the overheads of the three protocols. We define the overhead as the total bytes transmitted per successfully received bytes. There are two kinds of packets in these protocols, namely, Hello messages and data packets. As we can see, on average, the overhead of LSGO is about 8.62 greater than that of GyTAR and 5.85 greater than that of GpsrJ+. That is because LSGO selects a group of neighbor nodes to forward the packets in order to ensure VANET transmission reliability, at the same time increasing the overhead.

As shown in Figure 8, we compare the performance of the three protocols in terms of network throughputs. Overall, the network throughputs decrease with the increase in average vehicle velocity. In detail, the network throughput of GpsrJ + ranges from 640 to $469 \mathrm{kbps}$, while that of GyTAR decreases from 757 to 609 kbps. However, we find that the network throughput of GpsrJ + and GyTAR is always lower than that of LSGO. LSGO's throughput ranges from 1,650 to 1,430 kbps. LSGO can achieve about $855 \mathrm{kbps}$ and $1 \mathrm{Mbps}$ average gain compared with GyTAR and GpsrJ+. When the vehicle speed increases, the connected time between vehicles becomes shorter, and the link quality becomes poor. The possibility of appearing intermittent connectivity scenarios is higher. So, the three curves are all downward.

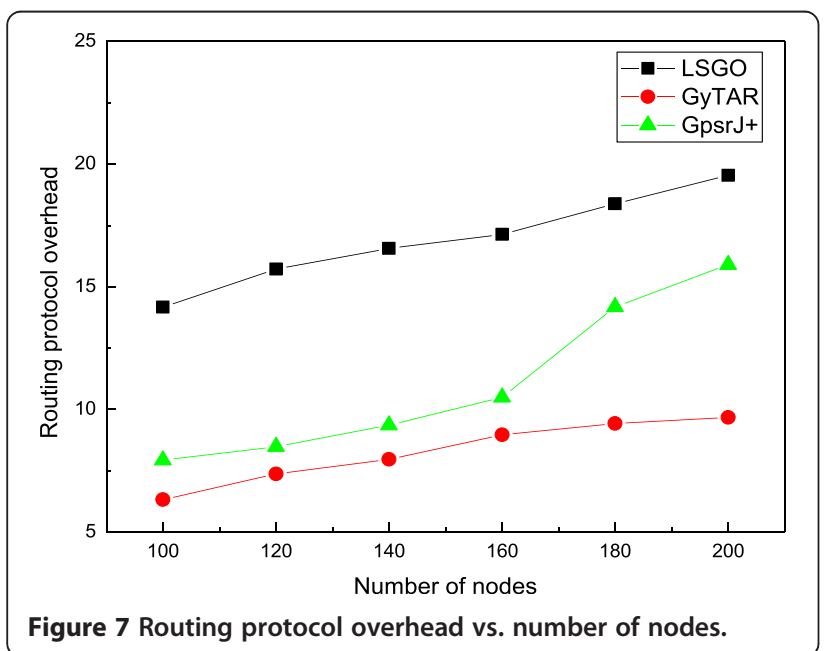

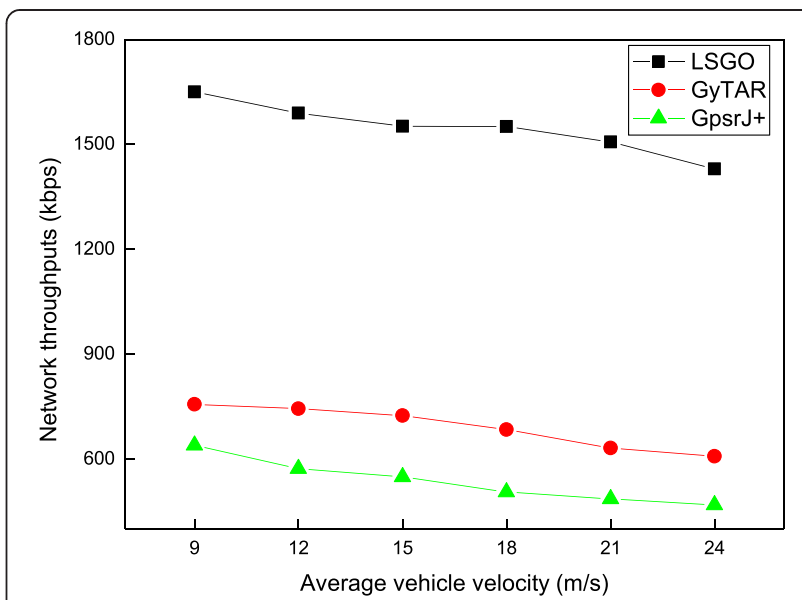

Figure 8 Network throughputs vs. average vehicle velocity.

LSGO selects a set of candidate nodes to forward the packet, so that it has a high probability of successful transmission of the packet, and consequently, the network throughput is improved.

Figure 9 illustrates the variation of the packet delivery ratio with the average vehicle velocity. We define the packet delivery ratio as the ratio of the number of packets successfully delivered to the destination to the number of the total packets generated in the simulation. As we can see, the average packet delivery ratio of LSGO is about $91.3 \%$, while that of GyTAR and GpsrJ + is about $69.3 \%$ and $71.3 \%$, respectively. The set of candidate nodes and the relative high-quality links used in LSGO contribute to the improvement. In LSGO, we improve the greedy forwarding strategy by taking the link state into account, so the nodes that have a bad-quality link have low priorities to forward the packet. Therefore, the packet delivery ratio achieved by GyTAR and GpsrJ + is lower than that by LSGO. As we can see, the tendency of the packet delivery ratio is upward at first

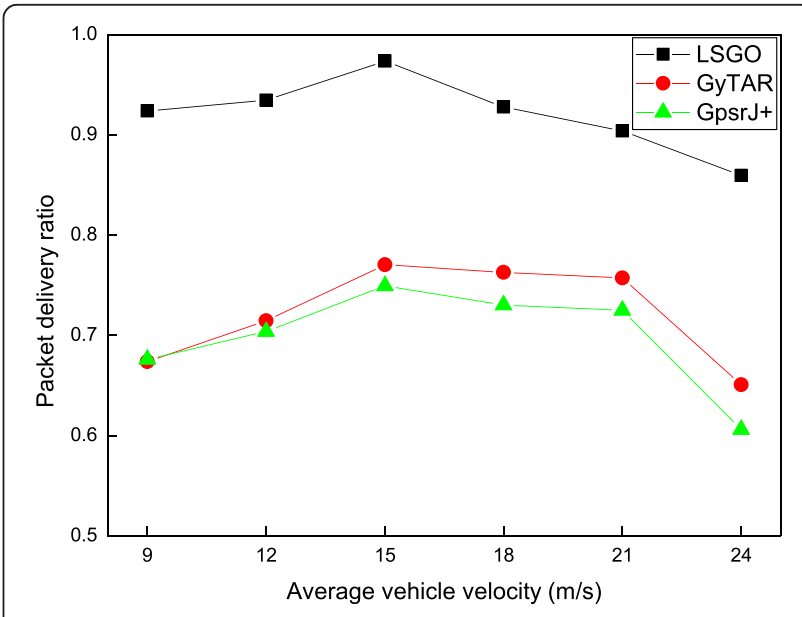

Figure 9 Packet delivery ratio vs. average vehicle velocity. 
and then drops with the increase in vehicle velocity. It is because that, for one node, the possibility of appearing effective neighbors becomes higher due to vehicles moving. However, when the vehicle speed continues to increase, the connectivity between vehicles deteriorates with this change, and the bad link quality results in a lower delivery ratio.

Figure 10 shows the performance of the end-to-end delay. We can find out a huge decline in the end-to-end delay of all these three protocols with increasing vehicle velocity, when the node number is fixed. The reason is that the time cost of carry and forward decreases with the increase in average vehicle velocity. We can learn that the average delay of GpsrJ + is nearly $0.04 \mathrm{~s}$. For GyTAR, the delay reaches $0.124 \mathrm{~s}$ when the average vehicle velocity is $9 \mathrm{~m} / \mathrm{s}$ and the minimum delay is $0.053 \mathrm{~s}$ when the average node speed is $24 \mathrm{~m} / \mathrm{s}$ in the network. The delay of LSGO achieves $0.016 \mathrm{~s}$ average gain compared with that of GyTAR. This superiority is due to the reason that LSGO uses opportunistic routing to ensure VANET transmission reliability, while reducing the number of transmissions. So, the time needed to retransmission is saved, and thus, the end-to-end delay is shortened, while in GyTAR, a considerable time would be wasted for retransmission.

Figure 11 reflects the overheads of the three protocols. As we can see, the routing protocol overhead increases with the increase in vehicle speed. It is because when the vehicle speed increases, the connectivity between vehicles becomes poor, which results in an increase in the number of Hello messages and increases the overhead. Overall, on average, the overhead of LSGO is about 16.3 greater than that of GyTAR and 11.7 greater than that of GpsrJ+. That is because LSGO uses a multicast mechanism and selects a group of neighbor nodes to forward the packets in order to ensure VANET transmission reliability, at the same time increasing the overhead. LSGO

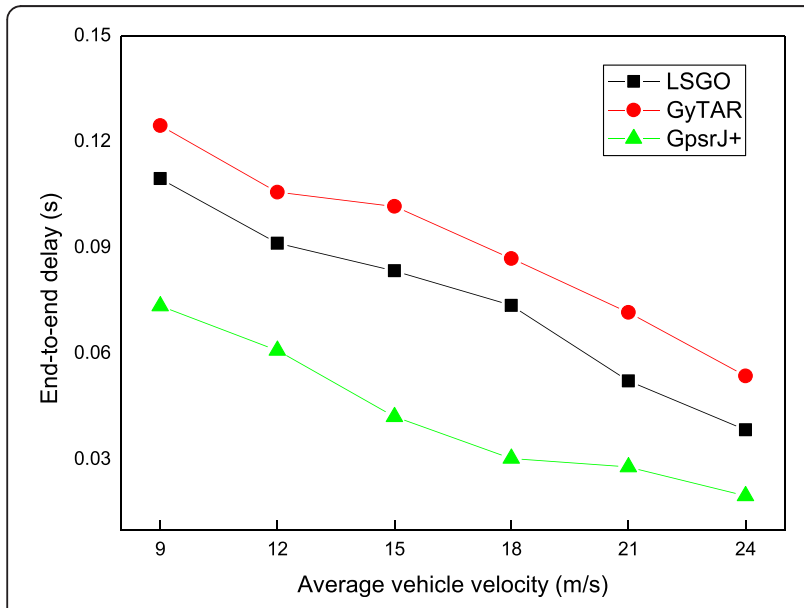

Figure 10 End-to-end delays vs. average vehicle velocity.

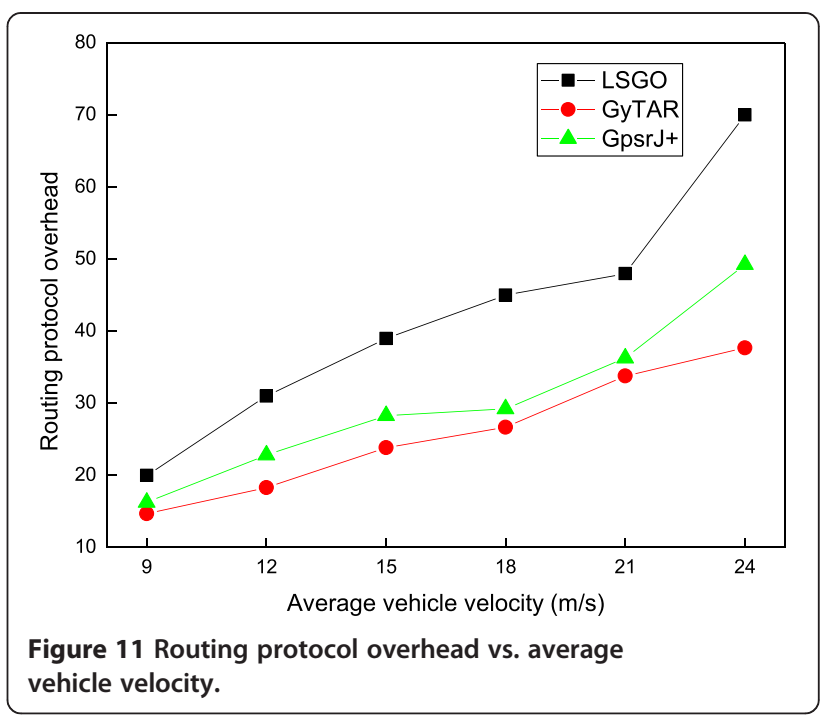

aims to improve the reliability of data transmission in a highly dynamic environment. In other words, we seek to improve the packet delivery rate. Therefore, we think that the cost of a little overhead in exchange for a higher delivery rate is reasonable.

\section{Conclusions}

In this paper, we put forward a new routing protocol for VANETs called Link State aware Geographic Opportunistic routing protocol (LSGO) which takes a combination of geographic location and the link state information as the forwarder selection mechanism. The protocol aims to ensure a highly dynamic network packet delivery rate and improve the reliability of data transmission. Besides, it also aims to reduce the number of transmissions (including retransmissions) and the transmission delay. LSGO uses an improved ETX mechanism to calculate the link transmission rate. The protocol is mainly composed of two parts: the candidate node set selection mechanism and the candidate node priority scheduling algorithm. To validate the performance of the protocol, we have compared it with GpsrJ + and GyTAR via ns-2. The simulation results showed that when the number of nodes changes, LSGO's packet dropping rate is reduced by about $28 \%$ and $17 \%$, and the network throughput is improved by about $42 \%$ and $22 \%$. When there are 100 vehicles in the network and the average vehicle velocity increases, LSGO's packet dropping rate is reduced by about $71.8 \%$ and $69.9 \%$, and the network throughput is improved by about $187 \%$ and $123 \%$. So, we can make a conclusion that LSGO achieves a higher throughput and lower packet dropping rate in highly dynamic networks. Although LSGO's overhead is slightly larger than that of the other two protocols, we think that the cost of a little overhead in exchange for a higher delivery rate is reasonable. 
In this paper, the theoretical analysis is less, and we only validate the performance of the protocol by simulation. So, in the future, we will do some theoretical analysis and further modify our routing protocol to reduce the overhead.

\section{Competing interests}

The authors declare that they have no competing interests.

\section{Acknowledgements}

This work was supported by the National Natural Science Foundation of China under Grant No. 61271176 and No. 61231008, the National Science and Technology Major Project under Grant No. 2011ZX03001-007-01 and No. 2013ZX03004007-003, the Fundamental Research Funds for the Central Universities, and the 111 Project (B08038).

\section{Received: 28 February 2014 Accepted: 29 May 2014}

Published: 17 June 2014

\section{References}

1. X Sun, X Li, Study of the feasibility of VANET and its routing protocols, in Proceedings of the International Conference on Wireless Communications, Networking and Mobile Computing, DaLian, October 2008, , pp. 1-4

2. $Y$ Lin, $Y$ Chen, $S$ Lee, Routing protocols in vehicular ad hoc networks: a survey and future perspectives. J. Inf. Sci. Eng. 26(3), 913-932 (2010)

3. CE Perkins, EM Royer, Ad-hoc on-demand distance vector routing, in Proceedings of the Sencond IEEE Workshop on Mobile Computing Systems and Applications (WMCSA'99), New Orleans, February 1999, pp. 90-100

4. V Naumov, R Baumann, T Gross, An evaluation of inter-vehicle ad hoc networks based on realistic vehicular traces, in Proceedings of the ACM MobiHoc'06 Conference, Florence, May 2006, pp. 108-119

5. F Li, Y Wang, Routing in vehicular ad hoc networks: a survey. IEEE Veh. Technol. Mag. 2(2), 12-22 (2007)

6. B Karp, HT Kung, GPSR: greedy perimeter stateless routing for wireless networks, in Proceedings of Mobile Computing and Net-working, Boston, August 2000, pp. 243-254

7. C Lochert, M Mauve, H Füssler, H Hartenstein, Geographic routing in city scenarios, in Proceedings of the SIGMOBILE Mobile Computing and Communications Review, vol. 9(1), 2005, pp. 69-72

8. KC Lee, J Haerri, U Lee, M Gerla, Enhanced perimeter routing for geographic forwarding protocols in urban vehicular scenarios, in Proceedings of the IEEE 2007 Globecom Workshops, Washington, DC, November 2007, pp. 1-10

9. V Naumov, TR Gross, Connectivity-aware routing (CAR) in vehicular ad-hoc networks, in Proceedings of the 26th IEEE International Conference on Computer Communications (INFOCOM'07), Anchorage, May 2007, pp. 1919-1927

10. S Schnaufer, W Effelsberg, Position-based unicast routing for city scenarios, in Proceedings of the 2008 International Symposium on a World of Wireless, Mobile and Multimedia Networks (WoWMoM 2008), Newport Beach, June 2008, pp. 1-8

11. M Jerbi, SM Senouci, Y Ghamri-Doudane, T Rasheed, Towards efficient geographic routing in urban vehicular networks. IEEE Trans. Veh. Technol. 58(9), 5048-5059 (2009)

12. K Shafiee, VCM Leung, Connectivity-aware minimum-delay geographic routing with vehicle tracking in VANETs. Ad Hoc Netw. 9(2), 131-141 (2011)

13. SH Cha, KW Lee, HS Cho, Grid-based predictive geographical routing for inter-vehicle communication in urban areas. Int. J. Distributed Sensor Networks 2012, 7 (2012)

14. $T$ Wu, $Y$ Wang, $W$ Lee, Mixing greedy and predictive approaches to improve geographic routing for VANET. Wirel. Commun. Mob. Comput. 12(4), 367-378 (2012)

15. $Y X u, W$ Li, Y Yang, Dynamic vehicle routing using an improved variable neighborhood search algorithm. J. Appl. Math. 2013, 12 (2013)

16. DSJ De Couto, D Aguayo, J Bicket, R Morris, A high throughput path metric for multi-hop wireless routing, in Proceedings of the 9th Annual International Conference on Mobile Computing and Networking (MobiCom' 03), San Diego, September 2003, pp. 134-146

17. K Miyakita, K Nakano, M Sengoku, S Shinoda, Evaluation of route expected transmission count of routing strategies in multi-hop wireless networks, in Int Symposium Nonlinear Theory Appl, 951-954 (Bologna, September 2006),
18. S Biswas, R Morris, ExOR: opportunistic multi-hop routing for wireless networks, in Proceedings of the 2005 Conference on Applications, Technologies, Architectures, and Protocols for Computer Communications, Philadelphia, August 2005, pp. 133-144

19. M Al-Rabayah, R Malaney, A new scalable hybrid routing protocol for VANETs. IEEE Trans. Veh. Technol. 61(6), 2625-2635 (2012)

20. Z Wang, Y Chen, C Li, Local cooperative relay for opportunistic data forwarding in mobile ad-hoc networks, in Proceedings of the IEEE International Conference on Communications (IICC2012), Ottawa, June 2012, pp. 5381-5386

21. K Miyakita, K Nakano, M Sengoku, S Shinoda, Theoretical analysis of route expected transmission count in multi-hop wireless networks. IEICE Trans. Commun. E91.B(8), 2533-2544 (2008)

22. C Lochert, H Hartenstein, J Tian, H Fussler, D Hermann, M Mauve, A routing strategy for vehicular ad hoc networks in city environments, in Proceedings of the IEEE Intelligent Vehicles Symposium (INS'03), Columbus, June 2003, pp. 156-161

23. S Chachulski, M Jennings, S Katti, D Katabi, Trading structure for randomness in wireless opportunistic routing, in Proceedings of the ACM Conference of the Special Interest Group on Data Communication (SIGCOMM), Kyoto, August 2007, pp. 169-180

24. J Zhao, G Cao, VADD: Vehicle-assisted data delivery in vehicular ad hoc networks, in Proceedings of the 25th IEEE International Conference on Computer Communications (IN-FOCOM 2006), Barcelona, April 2006, pp. 1-12

25. I Leontiadis, C Mascolo, GeOpps: geographical opportunistic routing for vehicular networks, in Proceedings of the IEEE International Symposium on World of Wireless, Mobile and Multimedia Networks, Helsinki, June 2007, pp. 1-6

26. X Mao, S Tang, X Xu, X Li, H Ma, Energy-efficient opportunistic routing in wireless sensor networks. IEEE. Trans. Parallel Distributed System 22(11), 1934-1942 (2011)

27. H Dubois-Ferriere, M Grossglauser, M Vetterli, Valuable detours: least-cost anypath routing. IEEE/ACM Trans. Networking 19(2), 333-346 (2011)

28. Z Wang, Y Chen, C Li, A novel cooperative opportunistic routing scheme in mobile ad hoc networks. IEEE. J. Selected Areas Commun. 30(2), 289-296 (2012)

29. AP Mazumdar, AS Sairam, TOAR: transmission-aware opportunistic ad hoc routing protocol. EURASIP. J. Wireless Commun. Networking 1, 1-19 (2013)

30. Y Li, A Mohaisen, Z Zhang, Trading optimality for scalability in large-scale opportunistic routing. IEEE Trans. Veh. Technol. 62(5), 2253-2263 (2013)

31. X Wang, W Huang, S Wang, J Zhang, C Hu, Delay and capacity tradeoff analysis for MotionCast. IEEE/ACM Trans. Networking 19(5), 1354-1367 (2011)

32. X Wang, L Fu, C Hu, Multicast performance with hierarchical cooperation. IEEE/ACM Trans. Networking 20(3), 917-930 (2012)

33. The Network Simulator - ns-2, The Network Simulator - ns-2. http://www.isi. edu/nsnam/ns/. Accessed 23 May 2013

34. J Haerri, F Filali, C Bonnet, M Fiore, VanetMobiSim: generating realistic mobility patterns for VANETs, in Proceedings of the ACM 3rd International Workshop on Vehicular Ad Hoc Networks (VANET'06), New York, September 2006, pp. 96-97

doi:10.1186/1687-1499-2014-96

Cite this article as: Cai et al: LSGO: Link State aware Geographic Opportunistic routing protocol for VANETs. EURASIP Journal on Wireless Communications and Networking 2014 2014:96.

\section{Submit your manuscript to a SpringerOpen ${ }^{\odot}$ journal and benefit from:}

- Convenient online submission

- Rigorous peer review

- Immediate publication on acceptance

- Open access: articles freely available online

- High visibility within the field

- Retaining the copyright to your article

Submit your next manuscript at $>$ springeropen.com 